\title{
Las comunidades indígenas como usuarios de la información
}

\author{
César Augusto Ramírez Velázquez *
}

Artículo recibido: 25 de abril de 2007.

Artículo aceptado:

31 de mayo de 2007.

\section{RESUMEN}

Se expone aquí cómo las comunidades indígenas, como cualquier otro sector de la sociedad, pueden ser consideradas usuarias de la información y por lo tanto presentan necesidades de información y un comportamiento informativo en la búsqueda de la información que necesitan. Es preciso comprender cuáles son las características informativas que presentan los miembros de dichas comunidades para que se pueda impulsar su desarrollo y, como ciudadanos, se les permita tener el acceso a la información ya que tienen derecho a éste. Se presentan algunas de estas reflexiones y algunos de los resultados de la investigación que se lleva a cabo con la comunidad tepehua ubicada en la Huasteca Hidalguense de México.

* Centro Universitario de Investigaciones Bibliotecológicas de la UNAM, México. carv@servidor.unam.mx

INVESTIGACIÓN BiBLIOTECOLÓGICA, Vol. 21, Núm. 43, julio/diciembre, 2007, México, ISSN: 0187-358X. pp. 209-230 
Palabras clave: Tepehuas; Usuarios de la información; Necesidades de información; Comunidades indígenas.

\begin{abstract}
Indigenous communities as information users

César Augusto Ramírez Velázquez

Indigenous communities as any other sector of society, are users of information and, therefore, have information needs and show specific information seeking. We must understand these characteristics in order to assist their development and as citizens exercise their right to information. In the present article these issues are reflected upon and results given of a research project in a Tepehua community of the Huasteca Hidalguense.
\end{abstract}

Keywords: Tepehua community; Information needs; Information users; Indigenous communities.

\title{
INTRODUCCIÓN
}

\begin{abstract}
$A$ unque los pueblos indígenas son los referentes culturales de las naciones Alatinoamericanas, resulta que son los menos beneficiados del progreso y los desarrollos y cambios sociales que haya tenido el país donde se encuentre inmersa la comunidad indígena.

Con respecto a lo anterior es preciso exponer las diferencias sociales, económicas y educativas que existen entre las comunidades indígenas y los centros urbanos. Además, es preciso reflexionar sobre las formas en las que el Estado moderno, intenta crear una nación o Estado nacionalista a costa de la desaparición de las comunidades indígenas, sin pensar en que las raíces de las naciones están precisamente en estos pueblos.
\end{abstract}

\section{CARACTERÍSTICAS GENERALES DE LAS COMUNIDADES INDÍGENAS}

Las grandes ciudades latinoamericanas tienen y atraen a los integrantes de las diversas comunidades indígenas que habitan cada uno de estos países; 
estas personas son calificadas con diversos sobrenombres despectivos, como lo indica Bonfil:

Cualquier rasgo que recuerde la estirpe original de la sociedad y la cultura mexicana, cualquier dato que ponga en evidencia el mundo indio presente en las ciudades, queda conjurado con el simple calificativo de naco. ${ }^{1}$

Ante esto, lo que la sociedad debe reflexionar es que la presencia de las comunidades indígenas y sus integrantes es evidente en toda la actividad de la nación, como lo pueden indicar los murales de muchos edificios de gobierno, los mercados públicos, los museos, así como el atesoramiento de algunos objetos en las casas de personas que poseen alguna muestra del trabajo donde se revela la importancia de lo indígena.

\section{Concepto de COMUNidAD INDÍGENA}

Las comunidades indígenas pueden ser consideras como aquellas que conservan la herencia y el origen de un país, y que a la vez son objeto de discriminación, desprecio social, marginación y olvido. Es decir, "el ser indígena es señal de la negación primera", ${ }^{2}$ y de la negación de todos los derechos humanos y ciudadanos.

Con la conquista de gran parte de los pueblos autóctonos de América Latina por parte de los españoles, la unión de la cultura colonizadora con la de las comunidades indígenas dio como resultado una comunidad con características diversas, muchas de ellas contradictorias, debido a la fusión violenta de dos identidades diferentes en su origen. Esta unión repercute en el presente y arrastra diversos aspectos anclados en el pasado. Es decir, la civilización española posee un rico legado de recuerdo mientras la indígena es heredera de los logros de las civilizaciones prehispánicas, por lo que se conjunta la presencia de lo tradicional con lo moderno, conjugando a la vez, elementos de ambas culturas en un intento de vivir juntas, haciendo valer la herencia recibida.

Por lo tanto, una comunidad indígena es aquella que concentra un legado cultural, ocupa un lugar en todo país; se identifica respecto del resto de la población porque habla un idioma distinto a la lengua oficial; y que además

1 Guillermo Bonfil Batalla, México profundo: una civilización negada, (México: Grijalbo, 1989), p. 89.

2 Arturo Jiménez, "Los términos indio e indígena ocultan a los pueblos reales: Montemayor", en La Jornada de en medio, 3 agosto 2000. 
tiene usos y costumbres distintas; y cuya organización política, social, cultural y económica se diferencia de los otros sectores sociales, porque se sostiene en sus costumbres.

Las comunidades indígenas cuentan con una identidad propia que deben defender en la nación o país en el que viven, ya que se sienten amenazadas en su identidad, porque su existencia en la sociedad en la que intentan desarrollarse se encuentra al margen de la misma.

Pero todo país está compuesto a su vez por dos elementos esenciales: las personas que los integran y el espacio geográfico con el que establecen un sentido de pertenencia, y que puede ser comunal, cuando es la tierra la principal fuente de subsistencia. Asimismo, aunado a dichos elementos, es necesario considerar las tradiciones indígenas, la historia, la cultura local, las costumbres, los hábitos de vida, la alimentación, así como las expresiones orales y monumentales, todo lo que aporta un sentido de identidad.

Un país viene siendo

la constitución de una nación, procura asociar de una manera política los intereses de un poder único, ya sea federal o central, con un orden jurídico y con una cultura única, aunque ésta pueda tener variantes sobre el mismo territorio, es decir, una asociación de ciudadanos constituida como un territorio determinado. ${ }^{3}$

\section{LA VISIÓN QUE SE TIENE DE LA COMUNIDAD INDÍGENA}

Al llegar los españoles a América, el territorio estaba dividido en regiones. Los primeros pobladores tenían sus propias características en cuanto a cultura, historia, lengua, estructura social, religión, etcétera, que innegablemente los distinguían de los colonizadores.

Actualmente se observa que los países con altos niveles de población indígena muestran diferencias, demasiado contrastantes, entre las comunidades indígenas y las comunidades urbanas, como puede verse, por ejemplo, en el Convenio núm. 169 de la OIT, el cual dice:

1. "El presente Convenio se aplica:

a) A los pueblos tribales en países independientes, cuyas condiciones sociales, culturales y económicas les distinga de otros sectores de la colectividad nacional, y que estén regidos total o parcialmente por sus propias costumbres o por una legislación especial; 
b) A los pueblos en países independientes, considerados indígenas por el hecho de descendencia de poblaciones que habitaban en el país o en una región geográfica a la que pertenece el país en la época de la Conquista o del establecimiento de las actuales fronteras estatales y que cualquiera que sea su situación jurídica, conservan todas sus propias instituciones sociales, económicas, culturales y políticas, o parte de ellas.

2. La conciencia de su identidad indígena o tribal deberá considerarse un criterio fundamental para determinar los grupos a los que se aplican las disposiciones del presente Convenio.

3. La utilización del término pueblos en este Convenio no deberá interpretarse en el sentido que tenga implicación alguna en lo que atañe a los derechos que pueda conferirse a dicho término en derecho internacional". ${ }^{4}$

Hemos tomado lo anterior como ejemplo de cómo se ven las comunidades indígenas, pero para efectos de este trabajo es conveniente retomar lo expresado por Rodríguez García (2001) en cuanto a las particularidades de cómo considerar a las comunidades indígenas:

a) Gran apego al territorio ancestral y los recursos naturales de su región.

b) Identidad propia e identificación como grupo cultural distinto.

c) Poseedores de una lengua indígena, comúnmente distinta a la lengua nacional.

d) Presencia de sus organizaciones sociales y políticas consuetudinarias, y

e) Producción principalmente orientada hacia la subsistencia.

Las anteriores características pueden explicar ciertos tipos de información y comportamientos informativos que tienen los integrantes de las comunidades indígenas, como se verá mas adelante.

\section{LA COMUNICACIÓN ORAL COMO FORMA DE TRANSMITIR} LA INFORMACIÓN EN LA COMUNIDAD

Para todo ser humano la forma más elemental de aprender es a través de los sonidos, la lengua. El primer contacto de aprendizaje es por medio de la palabra hablada, la cual se aprende de la madre, el padre y los familiares. Entre

4 Organización Internacional del Trabajo,"Convenio número 169 sobre pueblos indígenas y tribales en países independientes”, en América Indígena 58; 3.4 jul.-dic, 1996. 
más se comunica una persona, la lengua va creando esquemas de información y líneas de contacto con el mundo interno (la familia) y el externo, la comunidad.

Por eso, es preciso señalar que: "La historia o tradición oral se articula y trasmite por medio del idioma, sin embargo, se dice que la pérdida del idioma es la pérdida de la cultura". 5

La oralidad es el soporte de la palabra hablada, que puede ser tan vieja como el género humano; la transmisión de las culturas, incluyendo los mitos y leyendas, depende de cada lengua.

Por su parte, la escritura es un fenómeno relativamente reciente que ha revolucionado la forma de transmitir las ideas. Por citar un ejemplo, en el siglo XIX los intelectuales pusieron en duda la validez de la transmisión oral como medio de transmisión de las ideas de una generación a otra. Sin embargo, se puede constatar, que al estudiar las comunidades que carecen de un sistema de escritura (como ciertas comunidades indígenas de México), ésta carencia no es impedimento para la transmisión de ideas.

En el siglo XX, "los mismos hombres de letras desarrollaron métodos de análisis para aceptar las bases de los valores históricos que surgían de las fuentes orales"; ${ }^{6}$ estos métodos actualmente están sirviendo para crear bancos de datos en los que se rescata la herencia de la oralidad como medio de identidad regional y nacional.

En algunos pueblos indígenas todavía se mantienen las formas más tradicionales para transmitir los conocimientos, herencias y costumbres de la comunidad, como son:

los relatos y las fábulas, éstas son las más conocidas de las tradiciones; los mitos, las narraciones épicas y genealógicas; los proverbios, las adivinanzas y enigmas; las canciones y las conversaciones familiares. ${ }^{7}$

Como por ejemplo, las tribus de los pueblos africanos, se congregan en las noches alrededor de una hoguera y escuchan las leyendas que relata él o los miembros más ancianos.

En los pueblos mayas del sur de México, que colindan con Guatemala, los niños pequeños, quienes dependen totalmente del contacto familiar, son

5 John D. Waiko, "Safeguarding Papau New Guinean culture in the next millennium: educational reforms and preservation of cultural traditions", en Collecting and safeguarding the oral traditions: an international conference. Khon Kaen, Tha., 1999.

6 Ibidem

7 Mbathio Sall, "The importance of oral tradition for children: case of countries of the Sahel", en Collecting and safeguarding the oral tradition : an international conference, Khon Kaen, Tha., 1999 . 
orientados y educados por los bisabuelos, abuelos y padres, los cuales les enseñan en su lengua natal las actividades que deben desarrollar mientras crecen.

El propósito de la tradición oral reside en su concepción didáctica, es decir, "se extrae la esencia de los valores humanos, a través del conocimiento de la naturaleza, los comportamientos sociales y morales y de la creación de un héroe". 8

En los primeros años de vida de una persona, tanto en un pueblo indígena, como en una comunidad urbana, la oralidad es el medio más común de enseñanza y socialización, por lo que ésta puede interpretarse de la siguiente manera:

Es una fracción de nuestro tesoro nacional, el cual se integra con varios elementos: el primero debe entenderse como el paso para guiar y ayudar a determinar la sustancia de la identidad de la comunidad futura. Enseguida debe reconocerse que no falta mucho para destruir las tradiciones o sitios arqueológicos, en los cuales los daños son irreversibles y éstos no son propiedades culturales renovables. En las tradiciones y los sitios de nuestros antepasados se encuentran los valores intrínsecos de los cuales obtenemos valiosos datos informativos. Por último, existen valores culturales los cuales deben desarrollarse como bienes públicos rentables para el país 9

En otras palabras, la oralidad y la escritura deben considerarse como parte fundamental del rescate de las tradiciones y costumbres de las comunidades indígenas, con el fin de no perder las raíces históricas y culturales de cada grupo.

\section{NECESIDADES DE INFORMACIÓN Y SERVICIOS BIBLIOTECARIOS EN LAS COMUNIDADES INDÍGENAS}

En numerosas partes del mundo existen comunidades que cuentan con una gran tradición histórica y características culturales y educativas que condicionan el uso de su información. Al tratar de integrar esas comunidades al desarrollo de una nación, lo único que se hace es negar su origen y segregarlas de su participación en el Estado-nación. En este contexto, se debe pugnar por conocer sus necesidades informativas y su comportamiento informativo, ya que cuentan con patrones propios para generar, transmitir y difundir la 
información entre los miembros de la comunidad, con el fin de preservar su cultura y tradiciones o sus remanentes, ante el proceso civilizador dominante.

\section{LOS USUARIOS DE INFORMACIÓN EN COMUNIDADES INDÍGENAS}

Todo bibliotecario que vaya a planear algún servicio tiene, por principio, que identificar sistemáticamente los perfiles y necesidades de la comunidad usuaria de la información. Tanto el perfil de la comunidad como el del usuario permitirán identificar las características de la información que necesita la comunidad estudiada.

Para identificar el perfil de la comunidad se deben analizar:

a) Datos estadísticos de la población: tamaño, taza de crecimiento, rangos de edad, sexo, condición civil, religión, grupos étnicos, entre otros.

b) Información socio-económica: tipo de actividad, servicios comunitarios, facilidades recreativas, lugares de reunión.

c) Asuntos locales: reurbanización, desempleo, problemas de transporte.

d) Puntos de visita de los habitantes periódicos locales, radio local, grupos de acción, reuniones públicas, entre otros ${ }^{10}$.

Con relación a las necesidades de información es esencial identificar:

a) Nivel de experiencia sobre la disciplina de interés.

b) Tiempo de experiencia en la disciplina.

c) Si el usuario trabaja en forma individual o grupal.

d) Persistencia.

e) Motivación.

f) Capacidad de análisis de la información.

g) Conciencia del universo de recursos de información.

h) Medio ambiente en el que se desarrolla el usuario.

i) Manejo del lenguaje. ${ }^{11}$

Otra implicación importante de las necesidades de información es que éstas se refieren tanto a la obtención de la información como a los propósitos informativos del individuo. Objetivo que es condicionado por el estado psicológico de la persona y por el de los recursos informativos; es decir,

10 Alan Buch, The basic of information work (London: Clive Bingley, 1984): 17.

11 Patricia Hernández Salazar. La formación de usuarios de información en instituciones de educación superior (México: CUIB, 1998): 5 
el usuario no puede expresar sus necesidades de información, si no identifica éstas. Similarmente la satisfacción de éstas, es una medida de eficiencia de los sistemas de información encargados de resolver los problemas de información de los usuarios. ${ }^{12}$

Por otra parte, se dice que las necesidades de información son:

Carencias de conocimientos sobre un fenómeno, objeto, acontecimiento, acción o hechos que tiene una persona, producidos por factores externos e internos, que provocan un estado de insatisfacción, misma que el sujeto busca satisfacer a través de un comportamiento. ${ }^{13}$

Es preciso subrayar que los parámetros anteriores sirven sólo para transitar en un cierto tipo de comunidad y de usuarios, y no deberían ser presentados como el fin para crear categorías, tipologías de servicios y usuarios que puedan reflejar en la generalidad de la comunidad. Además, no se trata de ubicar a estos usuarios dentro de un patrón general. Así, cualquier actividad relacionada con la creación de servicios y la identificación de necesidades de información, invariablemente, se limitarán al fenómeno de los perfiles y necesidades de información.

De los dos aspectos anteriores se desprende la siguiente pregunta: ¿Cómo pueden identificarse el perfil y las necesidades de los usuarios de información en una comunidad indígena mexicana si se carece de estudios bibliotecológicos al respecto?

Al analizar las culturas indias con frecuencia es difícil establecer:

Los límites que separan lo económico de lo social: como difícil es distinguir lo que se cree, de lo que se sabe; el mito de la explicación y de la memoria histórica; el rito de los actos cuya eficiencia práctica ha sido comprobada una y otra vez, por generaciones. Por eso, junto a lo que llamaríamos un sólido conocimiento empírico, encontraremos prácticas rituales y creencias que llamaríamos mágicas, en un esfuerzo por ajustar la realidad cultural india a nuestra propia categoría aunque tales categorías en este caso, de origen occidental, no existan en esas culturas. ${ }^{14}$

Otro aspecto que podemos destacar de las comunidades indígenas es la cosmovisión, ésta es resultante de un conjunto cultural sobre el que cada

12 Richard L Drerr. "A conceptual analysis of information need", en Information Processing E Management, 19, 5 (1983): 276

13 Juan José Calva González, Análisis de las necesidades de información documental: teoría y método (Madrid: JJ Calva: 2001): 111-112

14 Guillermo Bonfil Batalla. México profundo: una civilización negada (México: Grijalbo, 1989): 55 
miembro del grupo posee un caudal de conocimientos en constante transformación, el cual se corrige, reproduce y sufre alteraciones durante el proceso de comunicación y actualización. En este sentido:

Se descubren dos características, al parecer antitéticas: por una parte, la similitud existente en las técnicas productivas, forma de organización social y política, concepciones acerca de la estructura social y política, concepciones acerca de la estructura del cosmos y otras prácticas, creencias e instituciones cuya semejanza deriva de una intensa y milenaria interacción; por otra, una riquísima diversidad en los campos señalados, que apuntan a la radical transformación histórica durante milenios de existencia mesoamericana, a una gran diversidad étnica y lingüística y a la variedad de climas y paisajes que fueron nichos ambientales de los pueblos indígenas. ${ }^{15}$

También se pueden encontrar algunos factores de similitud y diversidad:

Los primeros son los antecedentes históricos comunes, el desarrollo de un mismo contexto histórico y el predominio económico, político y cultural de unas sociedades sobre otras; los factores de diversidad son la influencia del medio en la concepción del cosmos, los distintos niveles de desarrollo social, las particularidades lingüísticas, la conformación histórica particular, la posición particular de las sociedades en las interrelaciones asimétricas de carácter económico, político, cultural y la construcción de su identidad. ${ }^{16}$

\section{Perfil de una comunidad indígena}

Para poder brindar un perfil de comunidad indígena desde la visión bibliotecológica, a continuación se hace una propuesta tomando como base los datos del XII Censo de Población, realizado por el INEGI, y se propone "la actualización, a nivel municipal, de la población indígena del país y por tanto conocer sus características socioeconómicas y demográficas" ${ }^{17}$ Esbozamos a continuación un perfil de este tipo de comunidades:

a) Datos estadísticos de la población. Hay 6044547 habitantes mexicanos que no hablan español, equivalentes al 7.13\% de la población nacional.

15 Alfredo López Austin, “El núcleo duro, la cosmovisión y la tradición mesoamericana”, en Cosmovisión, ritual e identidad de los pueblos indígenas de México (México: CNA: FCE, 2001): 49. 
El $93 \%$ de los indígenas se encuentra en trece entidades ubicadas en las regiones Centro, Sur y Sureste del país entre las que destacan Oaxaca, Chiapas, Veracruz, Yucatán y Puebla. Las lenguas indígenas de mayor habla son: náhuatl, maya, zapoteco, mixteco, otomí, tzetzatl y tzotzil. El $90.45 \%$ es población de 15 años y más que son alfabetos; el $91.32 \%$ es población de 6 a 14 años que asiste a la escuela; el $27.5 \%$ es población de 15 años y más con educación media superior y superior. El promedio de hijos por mujer, nacidos vivos es de $2.59 \%$, que se dividen entre fallecidos $0.28 \%$ y $2.31 \%$ de sobrevivientes.

b) Aspectos socioeconómicos. La población económicamente activa es del $49.33 \%$, porcentaje del cual se encuentra ocupado el $15.83 \%$ en el sector primario, el $27.82 \%$ en el secundario y el $53.35 \%$ en el terciario. La población ocupada que percibe más de dos salarios mínimos es del $43.56 \%$. Con relación a la vivienda, ésta suele ser un solo cuarto que incluye a la cocina. El promedio de ocupantes por vivienda es del $4.43 \%$ y el promedio de ocupantes por cuarto es de $1.2 \%$, en tanto que en las viviendas de $3 \%$ o más cuartos es del $71.24 \%$. En el rubro de servicios de la vivienda, se lee que el $88.76 \%$ tiene agua entubada dentro o fuera de ésta pero dentro del terreno, o que es una llave o hidrante públicos, o bien que la acarrean de otra vivienda. El 81.62\% ocupa gas para cocinar, y el $78.26 \%$ es propietario de la vivienda. La religión que se profesa en la mayoría de las comunidades es la católica, con el $87.99 \%$; el porcentaje restante se distribuye entre las religiones evangélica y protestante.

c) Productos locales. Una gran parte de las comunidades indígenas son pobres. Grandes extensiones de tierra no son aptas para cultivo y otras se han erosionado y producen menos porque han sido explotados de manera irracional. El desempleo está relacionado con la falta de productividad general de la tierra. La carencia de una industria con crecimiento equilibrado, hace que los productos nacionales no compitan con los extranjeros. En presencia de estas situaciones el indígena opta por vender su fuerza de trabajo en las grandes ciudades del país o el extranjero para tratar de aliviar la situación de sus familias. Por otra parte, las comunidades indígenas están pasando a formar parte de comunidades rurales, pues se comienzan a incorporar servicios urbanos como caminos rurales, telefonía rural, centros de salud, tiendas rurales, entre otros servicios más. Sin embargo, estas comunidades siguen siendo las más vulnerables dentro de la pobreza general del país.

d) Puntos de vista de los indígenas. Las asambleas, los comités y las faenas, así como la asistencia a las fiestas y reuniones religiosas son los puntos 
de reunión y en donde se difunde la información sobre el desarrollo de la comunidad. En algunas regiones del país se cuenta con radiodifusoras, las cuales dentro de su programa incluyen emisiones dirigidas a las comunidades en su lengua indígena. En otras, las menos, se llega a observar la circulación de periódicos, folletines y hojas sueltas en la lengua indígena, sólo que las notas son más de índole nacional que local.

\section{NECESIDADES DE INFORMACIÓN DEL USUARIO INDÍGENA}

Por falta de estudios sobre las necesidades de información de los indígenas, se proponen sólo generalidades, basadas en la investigación hecha en la Sierra Norte de Puebla ${ }^{18}$ y otras que surgieron de la observación directa en otras regiones del país. El acercamiento a las necesidades de información es el siguiente:

a) Nivel de experiencia del usuario sobre la disciplina de su interés. En estas comunidades se encuentran personas con dos tipos de formación: los monolingües y los biculturales. Los primeros son individuos que solamente se comunican en su lengua autóctona, la gran mayoría de ellos desarrollan su experiencia con base en la historia y tradición orales. Regularmente son adultos mayores (abuelos y bisabuelos). Los segundos, los biculturales son aquellos que además de comunicarse en su lengua indígena lo hacen en la segunda lengua que es el castellano, por consiguiente su panorama social traspasa las fronteras de su comunidad y región (aquí se ubican los niños, adolescentes, jóvenes y jóvenes-adultos). Cabe mencionar que, estos últimos, antes de ingresar a la educación formal de la escuela tuvieron que pasar por la formación tradicional de la familia y la comunidad. De este modo, el aprendizaje se pone a prueba desde la niñez y lo van reforzando los adultos, la madre o el abuelo, quienes por medio de una llamada de atención hacen que el niño aprenda.

Es preciso decir que uno de los rasgos que con mayor frecuencia llama la atención al estudiar la vida indígena

18 María del Rocío Graniel Parra, Edith Bautista Flores y Ariel A. Rodríguez García. "Acercamiento al perfil de recursos humanos para bibliotecarios rurales e indígenas: estudio de caso en la Sierra Norte de Puebla” (Encuentro Latinoamericano... 2001): 81. 
es el tratamiento benévolo y respetuoso que dan los padres a los hijos. Rara vez se educa mediante la violencia física y no se coarta la participación de los niños en la charla familiar. ${ }^{19}$

b) Tiempo de experiencia que tiene con la disciplina. Los indígenas monolingües y bilingües desarrollan su opinión en dos tenores: dentro de su cultura indígena y en aquella que se adquirió por añadidura, la cultura nacional. Los monolingües continúan conservando su cultura a pesar de las acometidas de la imposición de la cultura nacional. Los bilingües se dan a la tarea de mantener viva su cultura y aprender la segunda cultura con el propósito de hablar, leer y escribir unas cuantas palabras, las necesarias, para subsistir en el medio urbano. Ambas acciones se desarrollan a la vez para poder comunicarse con los miembros de su familia que no tuvieron oportunidad de asistir a la escuela.

c) Trabajo individual o grupal. En las comunidades indígenas la convivencia familiar y grupal es primordial, ya que la socialización del individuo comienza en la familia. Las normas sociales y culturales tienen rasgos comunes en los diversos estratos sociales, y entre niños, jóvenes, adultos y ancianos. Cada persona tiene diferentes funciones y tareas, por citar algún ejemplo: las mujeres están supeditadas a las decisiones y votos del esposo, aunque él no se encuentre en casa.

Por otra parte, el ámbito comunitario, como la asamblea o reunión comunitaria, es el medio que utilizan para difundir y tomar las decisiones. Los comités son los medios de gestión o administración de los bienes comunes. Las faenas, por su parte, son los mecanismos de cooperación activa esencialmente para la participación civil.

En el ámbito político se observa que los planes de desarrollo social del Gobierno Federal y Estatal, como son las tiendas comunitarias, las clínicas rurales, las escuelas, los caminos rurales, la dotación de energía eléctrica y telefonía, son aceptados rápidamente.

En el ámbito religioso hay una serie de cargos: Mayordomos, y Auxiliares como los Topiles y Fiscales, quienes permiten organizar las fiestas religiosas del patrono de la comunidad y, en su caso, el regional. También hay un grupo de danzantes, quienes se reúnen por invitación y herencia familiar. La organización más frecuente es por herencia y el familiar más viejo es el encargado de invitar a las nuevas generaciones a seguir con la tradición de la familia. 
d) Persistencia. La mayoría de los indígenas que han sido formados en la escuela insisten en conocer más sobre la cultura nacional que la local, debido a que la primera tiene más posibilidades de aprender lo que la segunda, la cual tiene como soporte de información la palabra hablada más que la escrita.

e) Motivación. Se encuentra por debajo del índice nacional debido a la ausencia de servicios bibliotecarios, además de las diferencias culturales, cognitivas y lingüísticas y al alto grado de estigmatización sobre su propia cultura y lengua. Se trata de personas que, al no depender tanto de lo escrito, tienden hacia lo oral como medio de acceso a la información.

f) Capacidad de análisis de la información. Como consecuencia de lo anterior, el lenguaje de saberes y conocimientos adquiridos es muy escaso a consecuencia de que lo aprendido está sustentado más en lo oral que en lo escrito. Es por eso que las características del análisis de la información contrastan con las necesidades de información y las posibilidades de vida de los indígenas.

g) Conciencia del universo de información sobre la disciplina. A pesar de que se cuenta con un amplio conocimiento sobre la diversidad de información en estos grupos, la falta de servicios bibliotecarios limita el acceso a ella. Por otra parte, el constante uso de la transmisión de ideas de forma oral, propicia que el indígena interprete el universo de la información de forma hablada más que escrita y que no valore este soporte de forma permanente.

h) Disponibilidad de recursos de información. Hay una escasez de recursos de información en las lenguas indígenas del país, a lo que se agrega la carencia de servicios bibliotecarios, pues al no haber atención a estas comunidades, no puede haber servicios por falta de materia prima: la información escrita en lengua indígena.

i) Medio ambiente en el que se desarrolla el usuario. Las comunidades indígenas viven en constante contacto con la naturaleza, y tienen un sentido de libertad y comunicación con ésta, lo cual le permite al indígena no atarse o esclavizarse al tiempo apresurado que caracteriza a las zonas urbanas. Es decir, coloca en el mismo plano de necesidad, actos de carácter aparentemente muy distintos, como por ejemplo, la selección adecuada de las semillas que debe sembrar, y hace una ceremonia propiciatoria para tener un buen tiempo. En otras palabras, busca obedecer los principios de orden universal, con los que el hombre se realiza y cumple su destino trascendente.

j) Manejo del lenguaje. Ante la presencia de representantes de nuevas ideas, costumbres o hábitos de vida, los padres de los niños indígenas 
les piden a sus hijos que no hablen la lengua de sus antepasados. Más bien les pide que se preparen dentro de la cultura y lengua oficial con el propósito de adaptarse a nuevas circunstancias, y que se apropien de aquellos elementos culturales ajenos que les sean útiles y compatibles para inventar nuevas soluciones, nuevas ideas, nuevas estrategias de acomodamiento que les permitan el acceso a un patrimonio cultural común, propio y distintivo.

Cada comunidad indígena mexicana posee perfiles comunitarios distintivos que son el resultado de una historia particular. La distribución territorial de esta población muestra una concentración mayor en las áreas que habían alcanzado un notable desarrollo cultural antes de la invasión europea.

Con base en lo anterior, y en el marco de una metodología social, se utilizó el impacto de la información en las comunidades indígenas; a través de las entrevistas personales de cada estrato de la comunidad tepehua se llevó a cabo la investigación y el análisis de los resultados sobre las necesidades informativas de dicha comunidad.

\section{DEMANDA DE LA INFORMACIÓN}

En las comunidades indígenas el uso de la información tiene como punto de referencia sus propias formas y los medios informativos. Las primeras surgen de la oralidad, como las pláticas familiares, las asambleas, los comités y las reuniones de los grupos comunitarios. Es decir, las fuentes de información son las mismas personas que transmiten los mensajes como medio de socialización de las ideas; por supuesto que la transmisión es a través de la lengua indígena.

Los medios informativos se dan cuando la oralidad, lengua indígena, es sustituida por la palabra escrita dispuesta en los libros, folletos y recursos audiovisuales como los casetes, videocasetes, la radio y la televisión. Con esto se inicia la intromisión de la segunda lengua, el castellano. De este modo, este tipo de medios es dirigido sobre todo a aquellos indígenas que han pasado por el proceso de educación formal ofrecido por la escuela oficial.

Ahora bien, las características de la comunicación indígena que intervienen en la demanda de información tienen que ver con los patrones o preferencias que prevalecen en el uso de información de cada población. Por citar algunos ejemplos: se puede encontrar que la información aparece en las conversaciones familiares y es transmitida de forma oral, o que los canales de comunicación suelen ser los padres y familiares, quienes se encargan de 
transmitir los mensajes en su lengua nativa, con el propósito de fomentar el uso de su idioma. Muy pocos se comunican en castellano.

Si algún miembro de la familia asiste a la escuela, tienen como fuente de información el libro de texto, donde la representación del conocimiento se da de manera escrita. Los maestros y compañeros de escuela son los refuerzos de su proceso de enseñanza-aprendizaje y existe un gran fomento al conocimiento, uso y abuso de otras culturas nacionales y extranjeras, pero no así de la propia.

Otras fuentes de información son las organizaciones sociales como las asambleas, los comités y las faenas, también las fiestas religiosas y la participación en algún grupo de danzantes o de músicos. La información se obtiene asistiendo a las reuniones comunitarias antes mencionadas, en las cuales se da aviso verbalmente sobre los asuntos que atañen a la comunidad. En esas reuniones se toman las decisiones sobre el futuro de la comunidad. En algunos casos los comunicados religiosos, públicos y comunitarios vienen en forma escrita, pues algún miembro de la familia sabe leer y escribir en castellano.

Otra cosa que permite identificar el uso y la demanda de la información, es el proceso de difusión. Éste se entiende como el espacio de tiempo, que luego de varias etapas o fases den origen a una decisión.

El primer acercamiento que tienen los niños indígenas para desarrollar su opinión es la conversación familiar. De acuerdo con el aprendizaje adquirido ellos ponen a prueba sus conocimientos, los cuales serán reforzados por los adultos, en este caso, la madre o el abuelo, quienes por medio de un regaño o corrección hacen que el niño aprenda.

El desarrollo de opinión de los adolescentes y los jóvenes indígenas se amplía con la convivencia comunitaria y la asistencia a la escuela. Sin embargo, hay aquí una contradicción en su formación, pues en estas edades surgen los interrogantes sobre su identidad e, incluso, la manifestación del rechazo de su origen; el cual es más bien reforzado por sus padres, quienes no desean que sus hijos sean maltratados y marginados por el sólo hecho de ser indígenas.

El factor que hace más notable esta especie de enmascaramiento mencionado, es la escuela, pues la incorporación del indígena al modelo educativo probado en una comunidad urbana, propicia en él la búsqueda de alternativas para salir de su comunidad y la experimentación de nuevos aprendizajes, lo que le resaltará su procedencia y resaltará sus problemas de pertenencia a su propia comunidad. Estas incursiones en la vida escolar y el contacto con la vida urbana fomentan con más vehemencia el uso de la segunda lengua, el castellano, dado que los libros de texto y los materiales didácticos están elaborados en esa lengua y no en la indígena.

Esta situación de enmascaramiento se agrava más cuando el individuo no encuentra expectativas de desarrollo social y económico en su comunidad, 
situaciones que lo obligan a abandonarla para conseguir los recursos económicos para él y su familia, lo que suele convertirlo en un emigrante, lo cual acelera su confundida identidad indígena.

Por otra parte, el desarrollo de la opinión de los adultos indígenas depende de dos vertientes: la lengua nativa y en castellano. El uso de la primera, le permite mantener vivo su idioma y poder conversar con los miembros de la familia que no tuvieron la oportunidad de asistir a la escuela, pero el uso del castellano más bien reprime sus valores culturales nativos.

Regularmente, aquellos que logran participar en el modelo educativo concluyen sus estudios, en el tercer grado de la educación primaria, y de este modo pasan a formar parte del grupo de analfabetos funcionales, pues sólo aprenden a leer y escribir unas cuantas palabras, las necesarias para subsistir en el medio urbano.

Además, quienes han tenido la oportunidad de emigrar del terruño logran desarrollar su opinión con base en el contacto cultural de la comunidad donde se colocaron para trabajar como peones, albañiles y trabajadoras domésticas, entre otros oficios, y al regresar a su comunidad emplean los conocimientos adquiridos para mejorar sus cultivos, para aumentar la calidad y diversidad de los productos, para mejorar sus viviendas o impulsar, con sus pláticas, la salida de más familias hacia esas experiencias de trabajo.

\section{Necesidades de información de la comunidad Tepehua. Resultados}

A continuación se presentan algunos resultados que fueron tomados de la investigación sobre necesidades de información en individuos de 12 a 16 años cuyo nivel de escolaridad es de secundaria en la comunidad indígena tepehua, radicada en la cabecera municipal de Huehuetla, Hidalgo, y que ejemplifican algunos aspectos tratados en este trabajo:

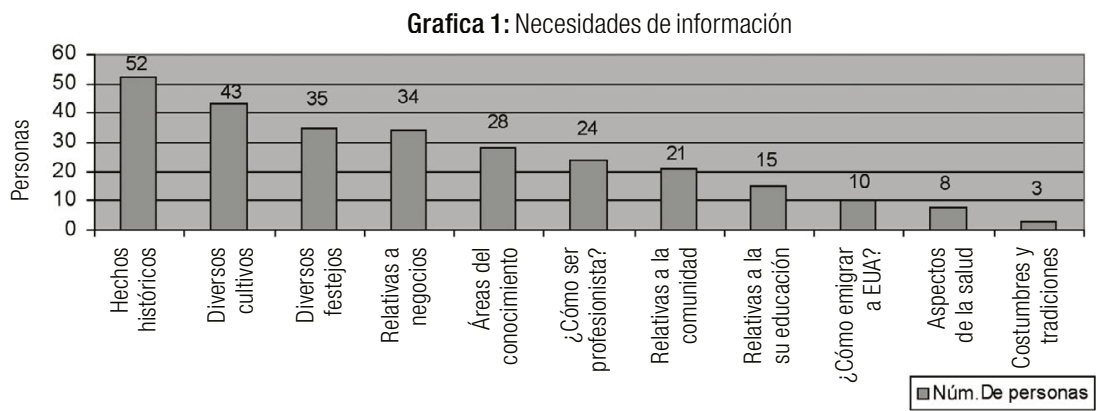


En la gráfica podemos observar que los individuos entrevistados, demuestran interés por diversos tópicos que, de acuerdo con la investigación realizada, nos dan una clara muestra de sus necesidades de información, las cuales se mencionan a continuación en orden de prioridad:

- Hechos históricos. Aquí se concentran varios tópicos relacionados con la historia de la humanidad y otros relacionados con la historia nacional: el descubrimiento de América, las guerras mundiales, la guerra de Irak; la independencia de México, la guerra de los pasteles, la batalla de Puebla, la Revolución Mexicana, la expropiación petrolera y el movimiento estudiantil de 1968.

- Diversos cultivos. De acuerdo con sus características, la mayor parte de la población se dedica al cultivo del café, sin embargo, por la baja tan drástica que tuvo éste a partir de 1998, su interés se enfoca ahora en conocer cómo se realizan otros cultivos, además del de café, como el de la caña, el del maíz, el del fríjol y el del cacahuate.

- Diversos festejos. Como en toda comunidad indígena existe un gran interés por saber por qué se celebran fechas importantes (desde festejos nacionales hasta específicos de la localidad), como por ejemplo: el 5 de febrero (día de la Constitución), el 24 de febrero (día de la bandera), el 18 de marzo (de la expropiación petrolera), el 21 de marzo (el natalicio de Benito Juárez y día de la primavera), semana santa (o todos santos como ellos la conocen), el 10 de junio (matanza de estudiantes por los "halcones", el 19 de septiembre (sismo en México en 1985), el 12 de octubre (día de la raza) y el 20 de noviembre (día de la Revolución Mexicana). Así como, "el baile del elote", que se acostumbra en el periodo de la cosecha, y el carnaval, que se realiza en conmemoración de la semana santa.

- Relativas a negocios. En la localidad de Huehuetla el comercio es uno de los principales generadores de empleo (en este sentido, existen alrededor de 30 comercios establecidos, entre tiendas, ferreterías, farmacias, papelerías, paleterías y establecimientos de comida). Pero también existen varios negocios familiares en las casas, en donde venden dulces y botanas, refrescos y cervezas, y algunos otros productos elaborados por las familias como el pan, las tortillas y el piloncillo. Los indígenas se interesaron en aprender asuntos relacionados con la elaboración del piloncillo, el café y el pan; o el costo de las mercancías, el cómo establecer un negocio (fotográfico o de venta de computadoras, una tienda, etcétera).

- Áreas del conocimiento. El nivel escolar en que se encuentran (secundaria) aunado a sus experiencias en la comunidad, ha derivado en que 
sus necesidades se enfoquen a diversas áreas temáticas como la agricultura, la ganadería, la pesca, la medicina, la ingeniería, la ecología, la economía, la política y el gobierno, y la administración de pequeños negocios.

- ¿Cómo ser profesionista? Este apartado es muy interesante, ya que nos indica que los jóvenes tepehuas han cambiado sus expectativas de desarrollo personal, y piensan en una formación profesional, entre las que destacan: ingeniero eléctrico, médico, enfermero, abogado, odontólogo y pedagogo; aunque algunos (los menos), consideran otras opciones como la de ser militar o electricista.

- Relativas a la comunidad. La percepción y la curiosidad de los jóvenes tepehuas en cuanto al conocimiento que tienen sobre los hechos que infieren del desarrollo de su comunidad y su participación en ella, se vislumbra a través de las siguientes inquietudes: ¿cuándo se hizo la carretera que llega a la localidad?, ¿cómo está organizada la comunidad?, ¿qué partidos políticos existen en el pueblo y cómo integrarse a ellos?, ¿cómo se compone el gobierno del pueblo?, ¿cómo respalda a la comunidad la Comisión de Derechos Humanos?, ¿cuáles son las costumbres y tradiciones de la comunidad tepehua?, ¿qué tanta contaminación se genera en la localidad?, ¿qué recursos forestales tiene Huehuetla y si existen leyes que regulen la tala de árboles en la zona?

- Relativas a su educación. Los jóvenes tepehuas tienen necesidad de saber acerca de algunos trámites escolares y de apoyos que pueden recibir por parte de las dependencias educativas. Algunas de sus necesidades de información son: saber cuánto se tarda uno en recibir el certificado, ¿cuándo se dan las becas de apoyo para sus estudios y qué se requiere para estudiar en la UNAM?

- Emigración a EUA. Uno de los aspectos que llaman la atención entre algunos jóvenes tepehuas es el de la emigración, pues debido a sus carencias económicas la consideran como una posibilidad para obtener mayores recursos para ellos y sus familias. Por eso su necesidad de información al respecto, es saber qué debe hacer para emigrar a los Estados Unidos (cabe hacer la acotación de que la información que se pide no se refiere a como hacerlo legalmente sino cómo podría hacerlo de manera ilegal; es decir: “¿cómo paso el río?”, “¿ cómo contacto a un pollero?” o “¿cómo le hago para que la migra no me detecte?”.

- Aspectos de la salud. Esto es algo que interesa particularmente en las siguientes direcciones: los medicamentos y las dosis que se le deben aplicar a un enfermo, ¿qué hacer para obtener una consulta en el Centro 
de Salud?, ¿qué vacunas se deben tener a cierta edad y cómo se puede llevar al hospital más próximo a un familiar?.

- Costumbres y tradiciones. Los jóvenes indígenas muestran interés en saber acerca de sus costumbres y tradiciones inquiriendo sobre los festejos que se realizan en su comunidad y porqué se realizan éstos, así como mostrando su interés en participar en ellos con preguntas como las siguientes: "¿cuándo y porqué se realiza 'la fiesta del elote’? ¿porqué nuestros abuelos nos cuentan historias del pueblo?; en Semana Santa se hacen muchos bailables, ¿quién los inventó y por qué?, ¿por qué se festeja la feria del café?; a mi me gustan mucho los bailes de las festividades pero, ¿qué significan?”.

Las necesidades de información anteriormente expresadas se refieren solamente a un estrato social de la comunidad tepehua (jóvenes con nivel secundaria); y por tanto, representan sólo una pequeña muestra de las diversas necesidades informativas que tienen las comunidades indígenas, ${ }^{20}$ las cuales deben ser detectadas teniendo como base un estudio sistemático de la comunidad, con una metodología acorde con el proceso científico y utilizando las técnicas y herramientas pertinentes.

\section{Conclusiones}

- En las comunidades indígenas, los métodos tradicionales de transmisión de las ideas, como la oralidad, no se han extinguido; sin embargo, el proceso de desarrollo de las nuevas generaciones de indígenas al obtener una nueva lengua (el castellano), muestra que están dejando de lado la oralidad para transmitir sus conocimientos e ideas cada vez más a través de la escritura. Aun así los bibliotecarios debemos de insistir en que la transmisión oral en las comunidades indígenas no debe perderse, pues de ese modo se conserva una tradición muy arraigada que, de no mantenerse, iría en perjuicio de la comunicación tan estrecha que tienen los individuos de las comunidades indígenas, sus familias y sus grupos sociales.

- La aseveración de que el panorama social de los indígenas bilingües los transporta más allá de las fronteras de su comunidad y región (aqui están los niños, adolescentes, jóvenes y jóvenes-adultos) se confirma, ya que

20 Ref. Aunque como ya se ha expresado, cada comunidad tiene sus propias necesidades de acuerdo a los factores externos e internos que las rodean. 
sus necesidades informativas se amplían conforme aumentan su aprendizaje en el aula y al basarse en textos en castellano adquieren una visión más amplia de lo que ocurre fuera del entorno de su comunidad.

- En relación al hecho de que los bilingües se dan a la tarea de mantener viva su cultura y aprender la segunda cultura con el propósito de bablar, leer y escribir unas cuantas palabras, las necesarias, para subsistir en el medio urbano, se puede aseverar, de acuerdo con el estudio presentado, que los jóvenes indígenas bilingües no solamente piensan en hablar, leer y escribir unas cuantas palabras, sino que se encuentran dispuestos a seguir desarrollando su nivel académico y a formarse en diversas actividades profesionales y con ello mejorar no sólo sus condiciones socioeconómicas particulares, sino también las de sus familiares y las de sus comunidades. Sin embargo muchos de ellos no pierden de vista la importancia de mantener y preservar su cultura y sus raíces, a través de los medios de transmisión de ideas y conocimientos que han utilizado comúnmente como la oralidad, pero también la escritura, ya cada vez más utilizada sobre todo por los jóvenes indígenas.

- Las comunidades indígenas forman un núcleo muy importante de habitantes en México, alrededor del 10\% de la población total y se encuentran asentadas (actualmente, debido a la migración) en la mayor parte del territorio nacional, por lo que es imprescindible conocer sus necesidades de información antes de planear y establecer bibliotecas y servicios adecuados para sus requerimientos informativos.

- Los servicios bibliotecarios que se planeen para apoyar a las comunidades indígenas deben contemplar colecciones documentales que estén de acuerdo con sus necesidades informativas en cuanto a las diversas temáticas que se vislumbren en los estudios de las diversas comunidades que para el caso se realicen, y habrá que tomar en cuenta todos los estratos de las personas que las compongan y que se pretenda atender.

- Por último se considera necesario hacer más investigaciones bibliotecológicas en el campo de las necesidades informativas de las comunidades indígenas y establecer servicios bibliotecarios que las atiendan, pues el fin debe ser apoyar su desarrollo y permanencia en este mundo globalizado y multicultural que ha tratado de absorberlas durante siglos en detrimento de sus tradiciones, historia, cultura, costumbres, hábitos de vida, alimentación y expresiones orales y monumentales; en pocas palabras la pérdida total de su identidad. 


\section{Bibliografía CONSULTADA}

Bonfil Batalla, Guillermo. México profundo: una civilización negada. México: Grijalbo, 1989, 250 p.

Buch, Alan. The basic of information work. London: Clive Bingley, 1984: 17.

Calva González, Juan José. Análisis de las necesidades de información documental: teoría y método. Madrid: JJ Calva: 2001: 111-112

Drerr, Richard L. "A conceptual analysis of information need", en: Information Processing E Management, 19, 5 (1983): 276

Fanon, Frantz. Los condenados de la tierra. México: FCE, 1961, 292 p.

Graniel Parra, María del Rocío, Edith Bautista Flores y Ariel A. Rodríguez García. "Acercamiento al perfil de recursos humanos para bibliotecarios rurales e indígenas: estudio de caso en la Sierra Norte de Puebla”. En: Encuentro Latinoamericano sobre la atención bibliotecaria a las comunidades indígenas. México: UNAM, CUIB; IFLA, 2001. p. 76-95.

Hernández Salazar, Patricia. La formación de usuarios de información en instituciones de educación superior. México: CUIB, 1998: 5

Jiménez, Arturo. "Los términos indio e indígena oculta a los pueblos reales: Montemayor". En: La Jornada de en medio, 3 agosto 2000.

López Austin, Alfredo. "El núcleo duro, la cosmovisión y la tradición mesoamericana". En: Cosmovisión, ritual e identidad de los pueblos indígenas de México. México: CNA: FCE, 2001. 49.

Organización Internacional del Trabajo. "Convenio número 169 sobre pueblos indígenas y tribales en países independientes”. En: América Indígena. 58; 3.4 jul.-dic, 1996.

Sall, Mbathio. "The importance of oral tradition for children: case of countries of the Sahel". En: Collecting and safeguarding the oral traditions: an international conference. Khon Kaen, Tha., 1999.

Valdés, Luz María. Los indios en los censos de población. México: UNAM, 1995. 273p.

Waiko, John D. "Safeguarding Papau New Guinean culture in the next millennium: educational reforms and preservation of cultural traditions". En: Collecting and safeguarding the oral traditions : an international conference. Khon Kaen, Tha., 1999. 